Article

\title{
Gini and Entropy based Spread Indexes for Primary Energy Consumption Efficiency and $\mathrm{CO}_{2}$ Emission
}

\author{
Hellinton H. Takada ${ }^{1 *}$, Celma O. Ribeiro ${ }^{1}$, Oswaldo L. V. Costa ${ }^{1}$ and Julio M. Stern ${ }^{2}$ \\ 1 Polytechnic School, University of São Paulo, São Paulo 05508-010, Brazil; \\ celma@usp.br (C.O.R.); oswaldo@lac.usp.br (O.L.V.C.) \\ 2 Institute of Mathematics and Statistics, University of São Paulo, São Paulo 05508-090, Brazil; \\ jstern@ime.usp.br (J.M.S.) \\ * Correspondence: hellinton@gmail.com
}

\begin{abstract}
Primary energy consumption is one of the key drivers of global $\mathrm{CO}_{2}$ emissions that, in turn, heavily depend on the efficiency of involved technologies. Either the improvement in technology efficiency or the expansion of non-fossil fuel consumption require large investments. The planning and financing of such investments, by policy makers or global energy firms, require, in turn, reliable measures of associated global spreads and their evolution in time. In this paper, our main contribution is the introduction of index measures for accessing global spreads (that is, measures of inequality or inhomogeneity in the statistical distribution of a related quantity of interest) of technology efficiency and $\mathrm{CO}_{2}$ emission in primary energy consumption. These indexes are based on the Gini index, as used in economical sciences, and generalized entropy measures. Regarding primary energy sources, we consider petroleum, coal, natural gas and non-fossil fuels. Between our findings, we attest some stable relations in the evolution of global spreads of technology efficiency and $\mathrm{CO}_{2}$ emission, and a positive relation between changes in global spreads of technology efficiency and use of non-fossil fuel.
\end{abstract}

Keywords: carbon emissions, energy consumption, technology efficiency, Gini index, generalized entropy index, fossil fuels, non-fossil fuels, petroleum, coal, natural gas

\section{Introduction}

Primary energy sources are those directly found in nature and usable without any human engineered transformation. Global consumption of primary energy has been steadily increasing throughout human history, see [1] for a good account of the last decade. Hence, the efficiencies, in terms of $\mathrm{CO}_{2}$ emission, of technologies used for primary energy consumption become key drivers of global $\mathrm{CO}_{2}$ release, [2]. There are different primary energy sources and several primary energy consumption technologies. Primary energy matrices and consumption technologies do vary, from country to country and along time. Our first main contribution in this paper is to define appropriate index measures of global spreads in efficiency, in terms of $\mathrm{CO}_{2}$ emissions, for technologies used in primary energy consumption.

Primary energy sources are classified as fossil and non-fossil fuels. Fossil fuels comprise crude oil or petroleum, natural gas and coal. These fuels come from remains of plants and animals buried millions of years ago and are, therefore, nonrenewable. Moreover the consumption of fossil fuels is responsible for most of the $\mathrm{CO}_{2}$ release due to human activities. In contrast, non-fossil fuels comprise hydropower, biomass and biofuels, wind, geothermal, solar, nuclear (fission and fusion), and so on. Non-fossil fuels are in general renewable, with a few exceptions like fission nuclear fuels. Consequently, the spread of non-fossil fuels is a most welcome factor in mankind's effort to reduce 
global $\mathrm{CO}_{2}$ emissions. Our second main contribution in this paper is to define appropriate index measures of global spreads in the use of non-fossil fuels.

Either the improvement of the primary energy consumption technology efficiency, in terms of $\mathrm{CO}_{2}$ emission, or the increase of the non-fossil fuel consumption, require significant infra-structure adaptations and large public and private investments. We believe that our proposed index measures of global spreads are useful tools in analysis and decision support that can benefit policy makers and global energy sector firms. Since the global demand for energy has been increasing over the last decades and will continue to grow, [3], long-term planning of investments in the energy matrix becomes of utmost importance, [4-10]. Our proposed index measures can provide global views and integrated perspectives of technology efficiency and non-fossil fuel use across different countries and the evolution of these scenarios over time.

In the context of economical sciences, the seminal work of Corrado Gini (1912) [11] introduced an income spread index measure referred in the literature as the Gini index. The Gini index measures the equality or inequality (homogeneity or inhomogeneity) of income distribution per capita. The Gini index has already been used to measure either the spread of the $\mathrm{CO}_{2}$ emissions per capita [12-15] or the spread of the energy consumption per capita [16]. In particular, Soares et al. (2018) [17] tried to compare the technology efficiency of different countries using clustering techniques based on the Gini index of $\mathrm{CO}_{2}$ emissions per capita, and subsequent comparative analyses of such clusters. In this paper, our approach is substantially different; it does not rely on group comparisons and, hence, does not depend on additional criteria used for intermediate cluster formation.

An alternative to the Gini index are entropy indexes conceived to generalize some spread measures like the mean log deviation, Atkinson index, Theil index, etc., see [18-21]. According to Bourguignon (1979) [19], a good spread index should have the property of additive decomposability, that is, it should provide a measure such that the total spread of a population can be broken down into a weighted average of spreads within subgroups of the same population. Unfortunately, the Gini index is not additive decomposable, motivating the construction of more suitable indexes in this respect, like generalized entropy indexes.

This paper is organized as follows: Section 2, presents details of our proposed methodologies to measure global spreads in efficiencies of technologies used for primary energy consumption, or to measure global spreads in use of non-fossil fuels. Section 3 presents an empirical study, describing the utilized data, and exemplifying the use of the Gini and the generalized entropy index. Section 4 gives our final conclusions.

\section{Methodologies}

In this section, we define the efficiency measures used in this paper for primary energy consumption technology. In addition, we present our proposed methodologies to measure global spreads of these quantities based on the Gini index and the generalized entropy index. We introduce the following notations: $e_{n, t}^{m}$ is the $\mathrm{CO}_{2}$ emission of country $n$, derived from consumption of primary energy source $m$, during time interval $t ; c_{n, t}^{m}$ is the consumption of country $n$, of primary energy $m$, during time interval $t ; n \in\{1, \ldots, N\} ; m \in\{1, \ldots, M\} ; t \in\{1, \ldots, T\} ; N$ is the number of countries, $M$ is the number of primary energies; and $T$ is the number of time intervals.

\subsection{Efficiencies in primary energy consumption technologies in terms of $\mathrm{CO}_{2}$ emissions}

We define the efficiencies in primary energy consumption technology in terms of $\mathrm{CO}_{2}$ emissions as follows:

$$
\varepsilon_{n, t}^{m}=\frac{c_{n, t}^{m}}{e_{n, t}^{m}}
$$

The technology efficiency $\varepsilon_{n, t}^{m}$ is an intensive variable and, consequently, it does not depend on countries' size, population, or gross domestic product. In contrast, the consumptions $c_{n, t}^{m}$ and the $\mathrm{CO}_{2}$ emissions $e_{n, t}^{m}$ are extensive variables, depending on countries' size. Notice that, for a fixed $e_{n, t}^{m}$, the 
technology efficiency $\varepsilon_{n, t}^{m}$ is higher when $c_{n, t}^{m}$ is higher and vice versa. For a fixed $c_{n, t}^{m}$, the technology efficiency $\varepsilon_{n, t}^{m}$ is higher when $e_{n, t}^{m}$ is lower and vice versa.

According to Lawrence et al. (2013), [16], quantities of interest concerning total energy consumption and $\mathrm{CO}_{2}$ emission are inextricably linked to equality/ inequality or homogeneity/ inhomogeneity measures related to the same quantities, and the study of these links constitutes one of the main motivations for the studies conducted in this paper. As far as we know, our study is the first, among similar studies, focusing primarily on the global probability density function, $f_{\varepsilon_{t}^{m}}(\cdot)$, and the global complementary cumulative distribution function, $\bar{F}_{\varepsilon_{t}^{m}}(\cdot)$, of technology efficiencies, $\varepsilon_{t}^{m}$.

By definition, the relationship between $\bar{F}_{\varepsilon_{t}^{m}}(\cdot)$ and $f_{\varepsilon_{t}^{m}}(\cdot)$ is

$$
\bar{F}_{\varepsilon_{t}^{m}}(\varepsilon)=\int_{\varepsilon}^{\infty} f_{\varepsilon_{t}^{m}}(z) d z
$$

The corresponding Lorenz curve is defined as a parametric bidimensional plot having

$$
x_{\varepsilon_{t}^{m}}(\varepsilon)=1-\bar{F}_{\varepsilon_{t}^{m}}(\varepsilon)=\int_{0}^{\varepsilon} f_{\varepsilon_{t}^{m}}(z) d z
$$

as its abscissa, and

$$
y_{\varepsilon_{t}^{m}}(\varepsilon)=\frac{1}{\left\langle\varepsilon_{t}^{m}\right\rangle} \int_{0}^{\varepsilon} z f_{\varepsilon_{t}^{m}}(z) d z
$$

as its ordinate. Moreover, $\varepsilon$ goes from 0 to $\infty$ and

$$
\left\langle\varepsilon_{t}^{m}\right\rangle=\int_{0}^{\infty} z f_{\varepsilon_{t}^{m}}(z) d z
$$

is the global technology efficiency for the consumption of the primary energy $m$ at the time interval $t$. This definition for the Lorenz curve is based on $[16,20]$. Notice that, since the values $x_{\varepsilon_{t}^{m}}(\varepsilon)$ and $y_{\varepsilon_{t}^{m}}(\varepsilon)$ are bounded between zero and one, the Lorenz curve always connects the points $(0,0)$ and $(1,1)$.

Our empirical estimator of the global complementary cumulative distribution function of the technology efficiency is given by

$$
\hat{\bar{F}}_{\varepsilon_{t}^{m}}\left(\tilde{\varepsilon}_{n, t}^{m}\right)=\frac{\sum_{i=n+1}^{N} \tilde{e}_{i, t}^{m}}{\sum_{i=1}^{N} \tilde{e}_{i, t}^{m}}, \forall n \in\{1, \ldots, N\},
$$

where the ordered set

$$
\left\{\tilde{\varepsilon}_{1, t}^{m}, \ldots, \tilde{\varepsilon}_{N, t}^{m}\right\}
$$

is obtained by sorting the set

$$
\left\{\varepsilon_{1, t}^{m}, \ldots, \varepsilon_{N, t}^{m}\right\}
$$

in the ascending order, and by the same country order applied to the sets

$$
\left\{e_{1, t}^{m}, \ldots, e_{N, t}^{m}\right\} \text { and }\left\{c_{1, t}^{m}, \ldots, c_{N, t}^{m}\right\}
$$

to obtain, respectively, the ordered sets

$$
\left\{\tilde{e}_{1, t}^{m}, \ldots, \tilde{e}_{N, t}^{m}\right\} \text { and }\left\{\tilde{c}_{1, t}^{m}, \ldots, \tilde{c}_{N, t}^{m}\right\}
$$

For instance, $\tilde{\varepsilon}_{1, t}^{m}=\min \left\{\varepsilon_{1, t}^{m} \ldots, \varepsilon_{N, t}^{m}\right\}$ and $\tilde{\varepsilon}_{N, t}^{m}=\max \left\{\varepsilon_{1, t}^{m} \ldots, \varepsilon_{N, t}^{m}\right\}$.

Since the empirical global technology efficiency for consumption of primary energy $m$ at time interval $t$ is

$$
\left\langle\hat{\varepsilon}_{t}^{m}\right\rangle=\frac{\sum_{i=1}^{N} \tilde{c}_{i, t}^{m}}{\sum_{i=1}^{N} \tilde{e}_{i, t}^{m}}=\frac{\sum_{i=1}^{N} c_{i, t}^{m}}{\sum_{i=1}^{N} e_{i, t}^{m}},
$$


it follows that

$$
\hat{x}_{\varepsilon_{t}^{m}}\left(\tilde{\varepsilon}_{n, t}^{m}\right)=\frac{\sum_{i=1}^{n} \tilde{e}_{i, t}^{m}}{\sum_{i=1}^{N} \tilde{e}_{i, t}^{m}} \text { and } \hat{y}_{\varepsilon_{t}^{m}}\left(\tilde{\varepsilon}_{n, t}^{m}\right)=\frac{\sum_{i=1}^{n} \tilde{c}_{i, t}^{m}}{\sum_{i=1}^{N} \tilde{c}_{i, t}^{m}}
$$

Using both $\hat{x}_{t}^{m}\left(\tilde{\varepsilon}_{n, t}^{m}\right)$ and $\hat{y}_{\varepsilon_{t}^{m}}\left(\tilde{\varepsilon}_{n, t}^{m}\right)$, it is possible to plot the corresponding empirical Lorenz curve.

The Gini index is a measure of statistical dispersion introduced by Corrado Gini in 1912 [11] to measure the income equality/inequality in a population. Using the Lorenz curve for technology efficiency, we define the Gini index of global spread of primary energy consumption technology efficiency, in terms of $\mathrm{CO}_{2}$ emissions, (short-named - technology efficiency Gini index) as follows:

$$
G_{\varepsilon_{t}^{m}}=\mathcal{A}_{\varepsilon_{t}^{m}} / \mathcal{B}_{\varepsilon_{t}^{m}}
$$

Considering the aforementioned $x_{\varepsilon_{t}^{m}}(\varepsilon)$ vs. $y_{\varepsilon_{t}^{m}}(\varepsilon)$ plot, $\mathcal{A}_{\varepsilon_{t}^{m}}$ is the area between the line connecting the points $(0,0)$ and $(1,1)$ and the Lorenz curve, and $\mathcal{B}_{\varepsilon_{t}^{m}}$ is the area of the triangle formed by the points $(0,0),(1,1)$ and $(0,1)$. Since $\mathcal{B}_{\varepsilon_{t}^{m}}=0.5$, it follows that $G_{\varepsilon_{t}^{m}}=2 \mathcal{A}_{\varepsilon_{t}^{m}}$. In addition, $0 \leq G_{\varepsilon_{t}^{m}} \leq 0.5$, with zero representing an equal global spread of the technology efficiency and 0.5 representing the highest possible inequality spread of the technology efficiency.

As an alternative to the Gini index, the generalized entropy index was introduced as a measure of income equality/inequality in a population [18]. We define the global spread of primary energy consumption technology efficiency index, in terms of $\mathrm{CO}_{2}$ emissions, based on the generalized entropy measure ( short-named - technology efficiency generalized entropy index) as

$$
E_{\varepsilon_{t}^{m}}^{\alpha}=\left\{\begin{array}{ll}
\frac{1}{N \alpha(\alpha-1)} \sum_{i=1}^{N}\left[\left(\frac{\varepsilon_{i, t}^{m}}{\bar{\varepsilon}_{t}^{m}}\right)^{\alpha}-1\right], & \alpha \notin\{0,1\}, \\
-\frac{1}{N} \sum_{i=1}^{N} \ln \frac{\varepsilon_{i, t}^{m}}{\bar{\varepsilon}_{t}^{m}}, & \alpha=0, \\
\frac{1}{N} \sum_{i=1}^{N} \frac{\varepsilon_{i, t}^{m}}{\bar{\varepsilon}_{t}^{m}} \ln \frac{\varepsilon_{i, t}^{m}}{\bar{\varepsilon}_{t}^{m}}, & \alpha=1,
\end{array} .\right.
$$

$\alpha$ is a real number and $\bar{\varepsilon}_{t}^{m}$ is the mean value of $\varepsilon_{i, t}^{m}, i=1, \ldots, N$. The values of $E_{\varepsilon_{t}^{m}}^{\alpha}$ go from 0 to $\infty$, with zero representing an equal global spread of the technology efficiency and higher values representing higher inequality of technology efficiency spread. The values $\alpha=0$ and $\alpha=1$ are of particular interest, namely, $E_{\varepsilon_{t}^{m}}^{0}$ is the technology efficiency mean log deviation index, and $E_{\varepsilon_{t}^{m}}^{1}$ is the technology efficiency Theil index.

The Atkinson index was introduced by Atkinson (1970) [21] and it can be derived from the generalized entropy index as follows. We define the global spread of the primary energy consumption technology efficiency based on the Atkinson index (short-named technology efficiency Atkinson index) as

$$
A_{\varepsilon_{t}^{m}}^{\epsilon}=\left\{\begin{array}{ll}
1-\frac{1}{\bar{\varepsilon}_{t}^{m}}\left[\frac{1}{N} \sum_{i=1}^{N}\left(\varepsilon_{i, t}^{m}\right)^{1-\epsilon}\right]^{1 /(1-\epsilon)}, & 0 \leq \epsilon \neq 1, \\
1-\frac{1}{\bar{\varepsilon}_{t}^{m}}\left(\prod_{i=1}^{N} \varepsilon_{i, t}^{m}\right)^{1 / N}, & \epsilon=1,
\end{array} .\right.
$$

$\epsilon$ is called the inequality aversion parameter, since $A_{\varepsilon_{t}^{m}}^{\epsilon}$ becomes more sensitive to changes at the lower end of the technology efficiency distribution as $\epsilon$ approaches 1, and $A_{\varepsilon_{t}^{m}}^{\epsilon}$ becomes more sensitive to changes in the upper end of the technology efficiency distribution as $\epsilon$ approaches 0 .

\subsection{Non-fossil fuel use}

The non-fossil fuel use of a country $n$ during the time interval $t$ is defined as follows:

$$
\rho_{n, t}=\frac{c_{n, t}^{\star}}{c_{n, t}}
$$

where $c_{n, t}^{\star}$ is the non-fossil fuel consumption of country $n$ during the time interval $t$ and $c_{n, t}$ is the total primary energy consumption of country $n$ during the time interval $n$, i.e. $c_{n, t}=\sum_{i=1}^{M} c_{n, t}^{i}$. Notice that 
the non-fossil fuel use $\rho_{n, t}$ is an intensive variable. As far as we know, our study is the first, among similar studies, focusing primarily on the global complementary cumulative distribution function $\bar{F}_{\rho_{t}}(\cdot)$ of the non-fossil fuel use, $\rho_{t}$.

We define the empirical estimator

$$
\hat{\bar{F}}_{\rho_{t}}\left(\tilde{\rho}_{n, t}\right)=\frac{\sum_{i=n+1}^{N} \tilde{c}_{i, t}}{\sum_{i=1}^{N} \tilde{c}_{i, t}}, \forall n \in\{1, \ldots, N\}
$$

where the ordered set

$$
\left\{\tilde{\rho}_{1, t}, \ldots, \tilde{\rho}_{N, t}\right\}
$$

is obtained sorting the set

$$
\left\{\rho_{1, t}, \ldots, \rho_{N, t}\right\}
$$

in the ascending order, using the same country order we obtain

$$
\begin{aligned}
& \left\{c_{1, t}, \ldots, c_{N, t}\right\} \text { and }\left\{c_{1, t}^{\star}, \ldots, c_{N, t}^{\star}\right\} \\
& \left\{\tilde{c}_{1, t}, \ldots, \tilde{c}_{N, t}\right\} \text { and }\left\{\tilde{c}_{1, t}^{\star}, \ldots, \tilde{c}_{N, t}^{\star}\right\} .
\end{aligned}
$$

Moreover, since the empirical global non-fossil fuel use at the time interval $t$ is

$$
\left\langle\hat{\rho}_{t}\right\rangle=\frac{\sum_{i=1}^{N} \tilde{c}_{i, t}^{\star}}{\sum_{i=1}^{N} \tilde{c}_{i, t}}=\frac{\sum_{i=1}^{N} c_{i, t}^{\star}}{\sum_{i=1}^{N} c_{i, t}},
$$

it follows that

$$
\hat{x}_{\rho_{t}}\left(\tilde{\rho}_{n, t}\right)=\frac{\sum_{i=1}^{n} \tilde{c}_{i, t}}{\sum_{i=1}^{N} \tilde{c}_{i, t}} \text { and } \hat{y}_{\rho_{t}}\left(\tilde{\rho}_{n, t}\right)=\frac{\sum_{i=1}^{n} \tilde{c}_{i, t}^{\star}}{\sum_{i=1}^{N} \tilde{c}_{i, t}^{\star}}
$$

Using both $\hat{x}_{\rho_{t}}\left(\tilde{\rho}_{n, t}\right)$ and $\hat{y}_{\rho_{t}}\left(\tilde{\rho}_{n, t}\right)$, it is possible to plot the corresponding empirical Lorenz curve.

Using the Lorenz curve for the non-fossil fuel use, we define the global spread of the non-fossil fuel use based on the Gini index (short-named - non-fossil fuel use Gini index) as

$$
G_{\rho_{t}}=\mathcal{A}_{\rho_{t}} / \mathcal{B}_{\rho_{t}}
$$

Considering the $x_{\rho_{t}}(\rho)$ vs. $y_{\rho_{t}^{m}}(\rho)$ plot, $\mathcal{A}_{\rho_{t}^{m}}$ is the area between the line connecting the points $(0,0)$ and $(1,1)$ and the corresponding Lorenz curve, and $\mathcal{B}_{\rho_{t}}$ is the area of the triangle formed by the points $(0,0),(1,1)$ and $(0,1)$. Since $\mathcal{B}_{\rho_{t}}=0.5$, it follows that $G_{\rho_{t}}=2 \mathcal{A}_{\rho_{t}}$. Moreover, $0 \leq G_{\rho_{t}} \leq 0.5$, with zero representing a homogeneous global spread of the non-fossil fuel use, and 0.5 representing the most inhomogeneous spread possible for non-fossil fuel use.

Analogously, for the technology efficiency generalized entropy index, we define the global spread of non-fossil fuel use based on the generalized entropy index or (short-named - non-fossil fuel generalized entropy index) as

$$
E_{\rho_{t}}^{\alpha}=\left\{\begin{array}{ll}
\frac{1}{N \alpha(\alpha-1)} \sum_{i=1}^{N}\left[\left(\frac{\rho_{i, t}}{\bar{\rho}_{t}}\right)^{\alpha}-1\right], & \alpha \notin\{0,1\}, \\
-\frac{1}{N} \sum_{i=1}^{N} \ln \frac{\rho_{i, t}}{\bar{\rho}_{t}}, & \alpha=0, \\
\frac{1}{N} \sum_{i=1}^{N} \frac{\rho_{i, t}}{\bar{\rho}_{t}} \ln \frac{\rho_{i, t}}{\bar{\rho}_{t}}, & \alpha=1,
\end{array} .\right.
$$

$\alpha$ is a real number, and $\bar{\rho}_{t}$ is the mean value of $\rho_{i, t}, i=1, \ldots, N$. The values of $E_{\rho_{t}}^{\alpha}$ go from 0 to $\infty$, with zero representing an equal global spread of non-fossil fuel use, and higher values representing higher inequality spreads of non-fossil fuel use. Of particular interest are the values $\alpha=0$ and $\alpha=1$ : $E_{\rho_{t}}^{0}$ is the non-fossil fuel mean $\log$ deviation index and $E_{\rho_{t}}^{1}$ is the non-fossil fuel Theil index. 




(a)

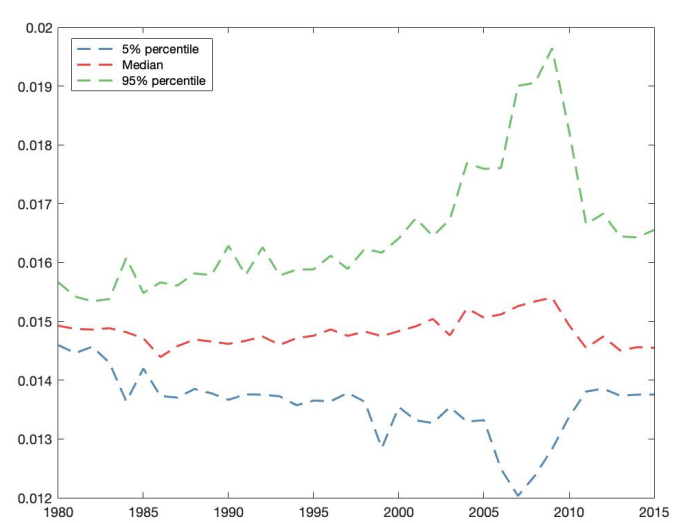

(b)

Figure 1. Petroleum consumption technology efficiency in terms of $\mathrm{CO}_{2}$ emissions for (a) a sample of countries and (b) the set's median, $5 \%$ percentile and $95 \%$ percentiles.

Analogously, for the technology efficiency Atkinson index, we define the non-fossil fuel Atkinson index as

$$
A_{\rho_{t}}^{\epsilon}=\left\{\begin{array}{ll}
1-\frac{1}{\bar{\rho}_{t}}\left[\frac{1}{N} \sum_{i=1}^{N}\left(\rho_{i, t}\right)^{1-\epsilon}\right]^{1 /(1-\epsilon)}, & 0 \leq \epsilon \neq 1, \\
1-\frac{1}{\bar{\rho}_{t}}\left(\prod_{i=1}^{N} \rho_{i, t}\right)^{1 / N}, & \epsilon=1,
\end{array} .\right.
$$

The scalar $\epsilon$ is known as the inequality aversion parameter, since $A_{\rho_{t}}^{\epsilon}$ becomes more sensitive to changes at the lower end of the non-fossil fuel use distribution as $\epsilon$ approaches 1 , and $A_{\rho_{t}}^{\epsilon}$ becomes more sensitive to changes in the upper end of the non-fossil fuel use distribution as $\epsilon$ approaches 0 .

\section{Empirical studies}

This section illustrates the use of our proposed methodology using the US Energy Information Administration (EIA) data [22] from 1980 to 2015, for a set of 41 countries (Albania, Argentina, Australia, Austria, Bangladesh, Belgium, Brazil, Bulgaria, Burma, Canada, Chile, China, Colombia, Egypt, Finland, France, Hungary, India, Indonesia, Iran, Ireland, Italy, Japan, Luxembourg, Malaysia, Mexico, Morocco, Netherlands, New Zealand, Nigeria, Norway, Pakistan, Peru, Romania, Spain, Switzerland, Taiwan, Tunisia, United Kingdom, United States, Venezuela). Countries were selected using criteria of data availability for the time period onder consideration. Consumptions and emissions data are in quad-BTU and MM Tons of $\mathrm{CO}_{2}$, respectively.

\subsection{Consumption technology efficiency in terms of $\mathrm{CO}_{2}$ emissions}

Regarding fossil primary energy sources, we consider petroleum, coal and natural gas. We present the consumption technology efficiency in terms of $\mathrm{CO}_{2}$ emissions along time in Figures 1, 2, 3 and 4 for petroleum, coal, natural gas and fossil fuel, respectively. For each case, we present technology efficiencies for a sample of countries and also the median, $5 \%$ percentile and $95 \%$ percentile of the entire set of considered countries. Notice that, while the technology efficiency medians are relatively stable along time, the percentiles have different behaviors. The distance between the percentiles and the medians gives an intuitive idea of the global spread of technology efficiency along time.

In order to measure the global spread of technology efficiency, we first use the technology efficiency Gini index, computed from the corresponding Lorenz curve; and these are present in Figures 5, 6, 7 and 8, for petroleum, coal, natural gas and fossil fuels. For each case, we present the Lorenz curve for two dates: 1980 and 2015. In addition, we also indicate some of the countries in the figures for illustration purposes. Since the obtained Lorenz curves are very close to the diagonal line, we conclude that technology efficiency is nearly equally distributed between countries. 


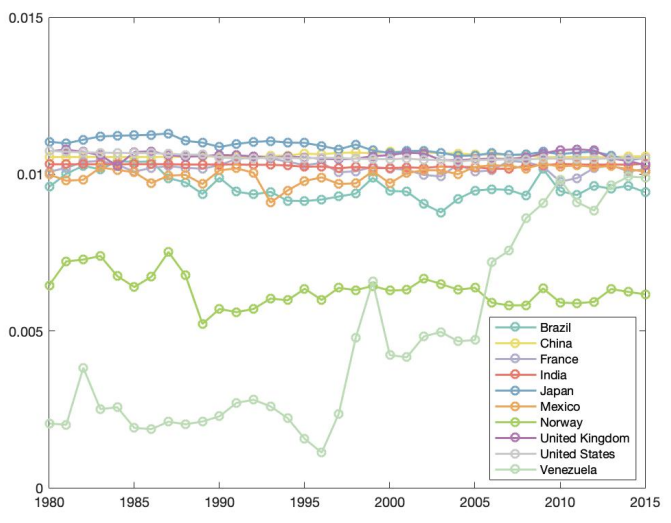

(a)

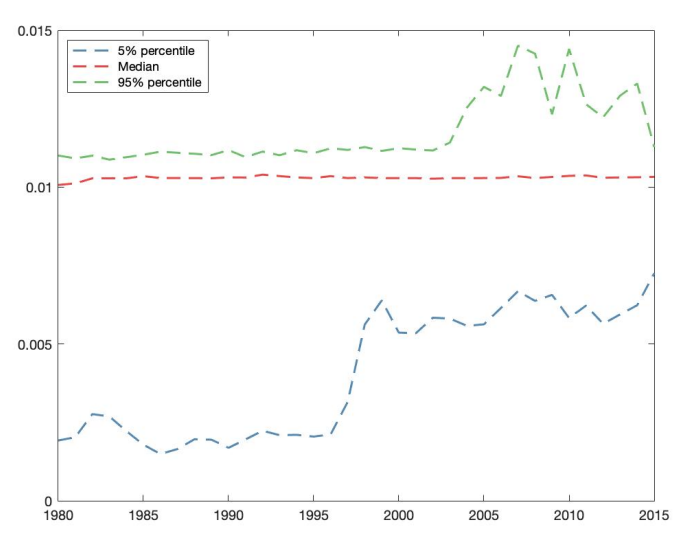

(b)

Figure 2. Coal consumption technology efficiency in terms of $\mathrm{CO}_{2}$ emissions for (a) a sample of countries and (b) the set's median, $5 \%$ percentile and $95 \%$ percentile.

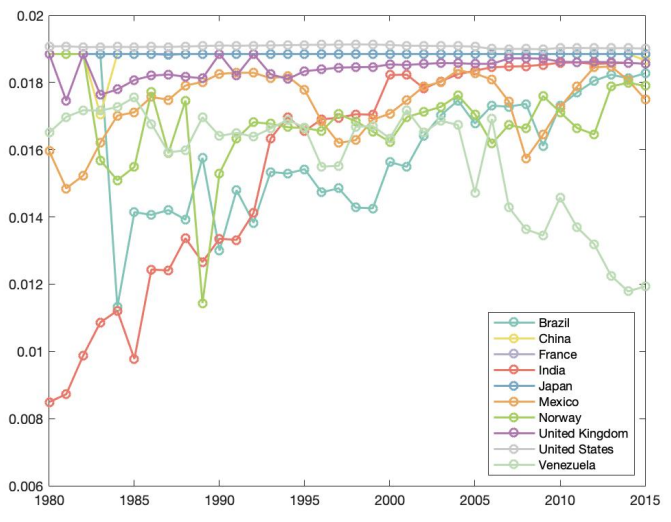

(a)

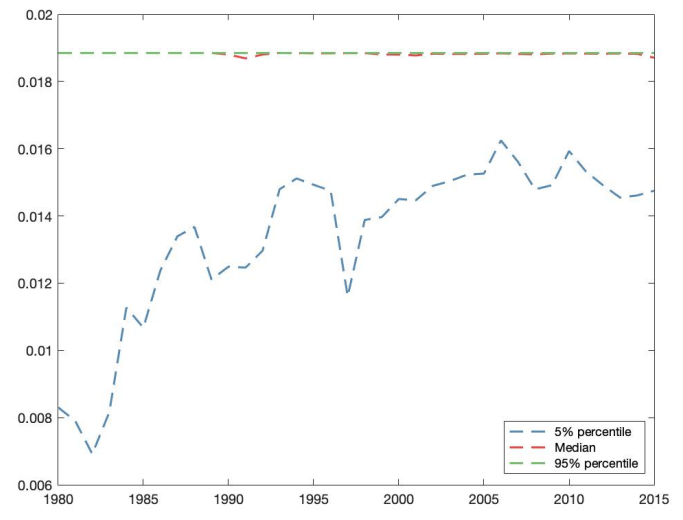

(b)

Figure 3. Natural gas consumption technology efficiency in terms of $\mathrm{CO}_{2}$ emissions for (a) a sample of countries and (b) the set's median, $5 \%$ percentile and the $95 \%$ percentile.

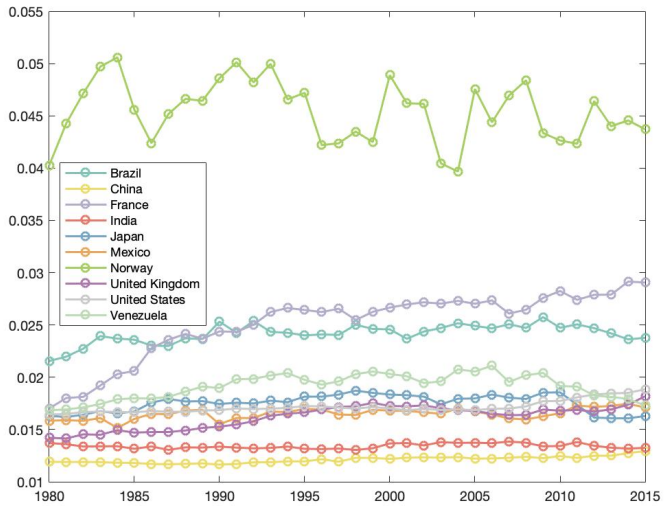

(a)

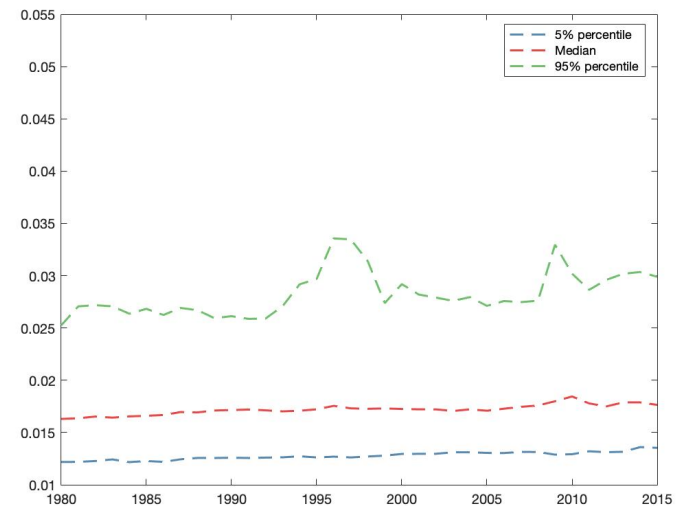

(b)

Figure 4. Fossil fuels consumption technology efficiency in terms of $\mathrm{CO}_{2}$ emissions for (a) a sample of countries and (b) the set's median, $5 \%$ percentile and $95 \%$ percentile. 


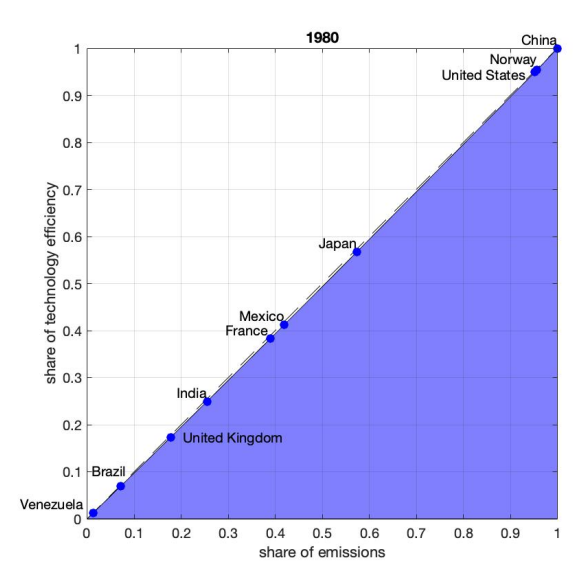

(a)

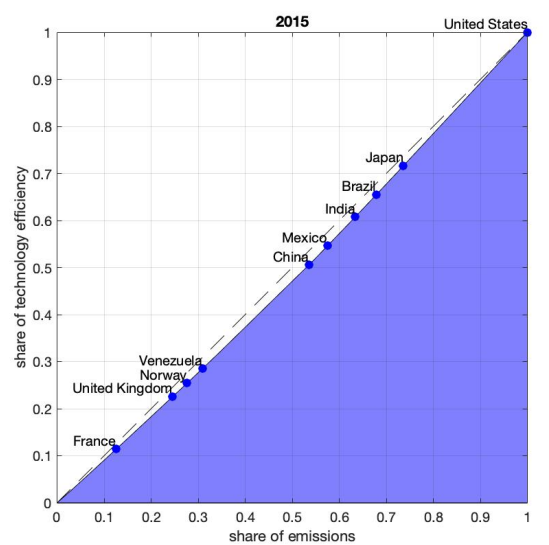

(b)

Figure 5. Lorenz curve of the petroleum consumption technology efficiency in terms of $\mathrm{CO}_{2}$ emissions in (a) 1980 and (b) 2015.



(a)

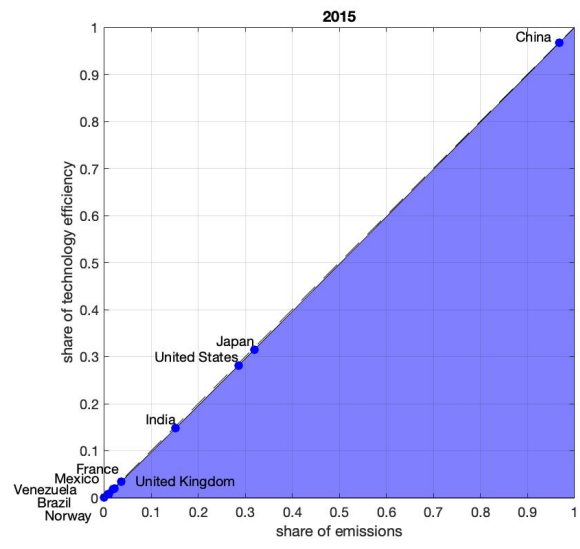

(b)

Figure 6. Lorenz curve of the coal consumption technology efficiency in terms of $\mathrm{CO}_{2}$ emissions in (a) 1980 and (b) 2015.

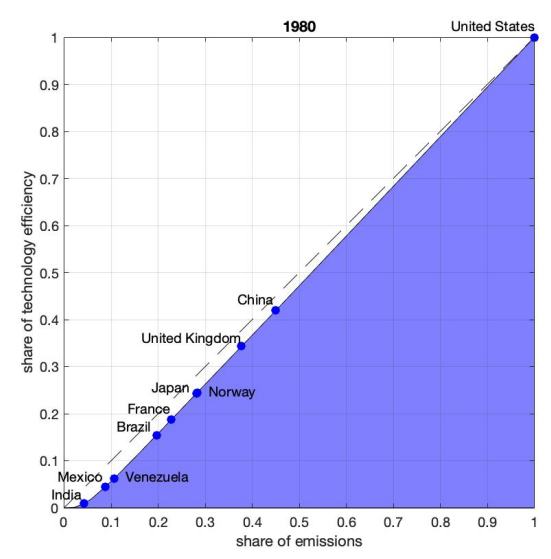

(a)

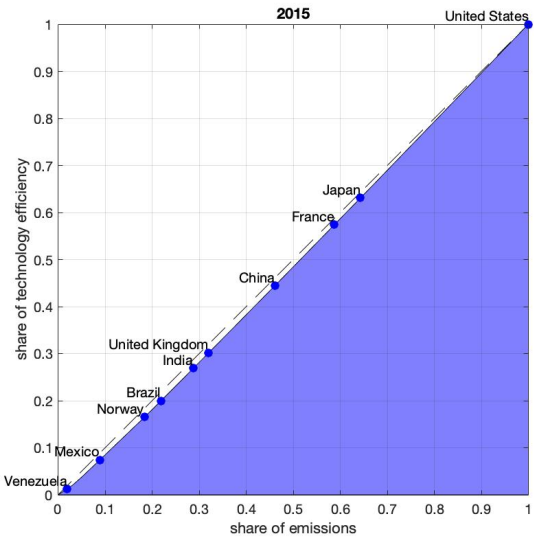

(b)

Figure 7. Lorenz curve of the natural gas consumption technology efficiency in terms of $\mathrm{CO}_{2}$ emissions in (a) 1980 and (b) 2015. 




(a)

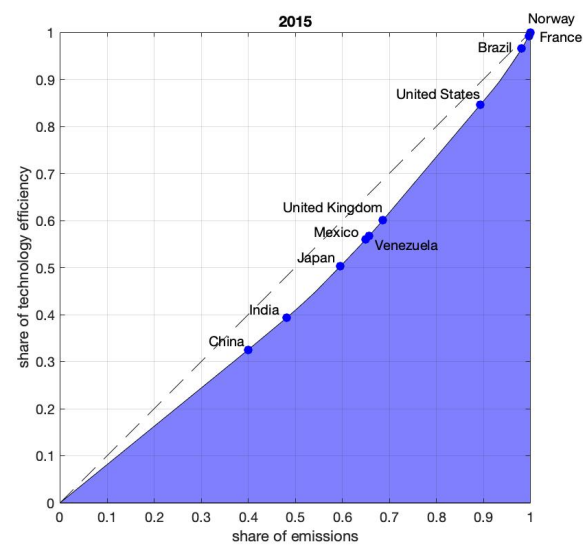

(b)

Figure 8. Lorenz curve of the fossil fuels consumption technology efficiency in terms of $\mathrm{CO}_{2}$ emissions in (a) 1980 and (b) 2015.

Using technology efficiency Lorenz curves for each year, we obtain evolution along time of this index. In Figure 9, we present the technology efficiency Gini indexes for petroleum, coal, natural gas and fossil fuels. The obtained Gini indexes are numerically close to zero along time, indicating technology efficiencies nearly equally distributed between the countries. Moreover, while the equality of the technology efficiency for coal and natural gas between 1980 and 2015 seems to be improving, the opposite seems to occur for petroleum and fossil fuels. Fossil fuels are composed by petroleum, coal and natural gas. However, since the Gini index does not satisfy the additive decomposability, it is not easy to grasp the relation between these those indexes.

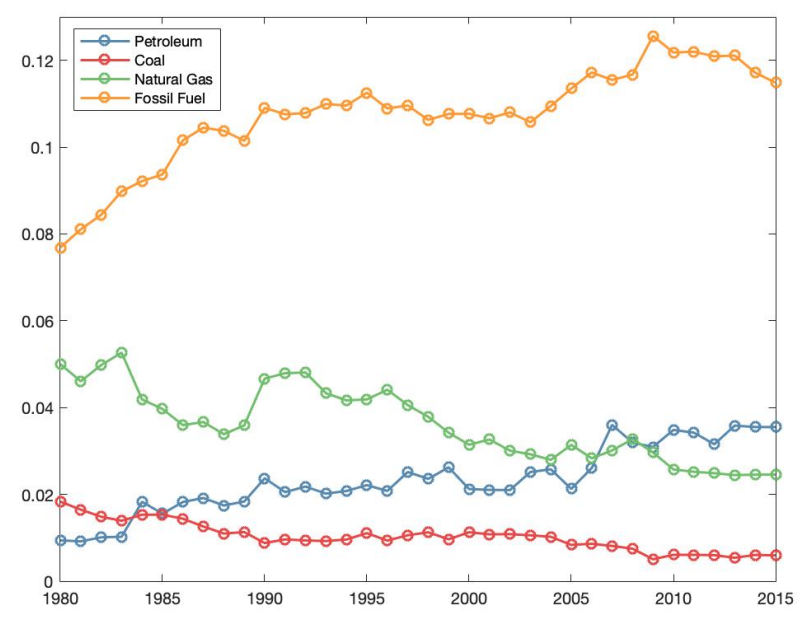

Figure 9. Technology efficiency Gini indexes for petroleum, coal, natural gas and fossil fuels.

In Figure 10a, we present the evolution along time of empirical global technology efficiency for consumption of petroleum, coal, natural gas and fossil fuels. It is possible to notice that the global technology efficiency for the consumption of coal is stable along time while the global technology efficiencies for the consumption of petroleum and natural gas seem to slightly increase along time. During the considered time period, the global technology efficiency is highest for natural gas, and lowest for coal. In Figure 10b, we present the mean value, among countries, for the technology efficiency for the consumption of petroleum, coal, natural gas and fossil fuels. Notice that the relative positions of countries for technology efficiency in fossil fuels consumption is (almost) the same in several of these plots. 


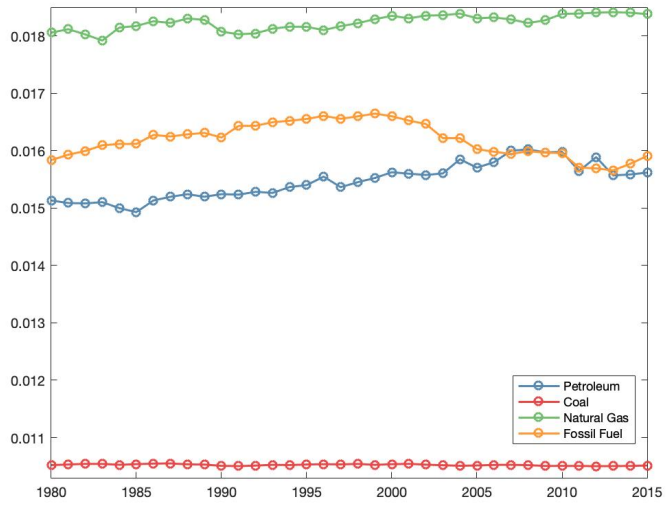

(a)



(b)

Figure 10. (a) The empirical global technology efficiency for the consumption of petroleum, coal, natural gas and fossil fuel along time, $\left\langle\hat{\varepsilon}_{t}^{m}\right\rangle$. (b) The mean between the countries of the technology efficiency for the consumption of petroleum, coal, natural gas and fossil fuel along time, $\bar{\varepsilon}_{t}^{m}$.

In Figure 11, we present the technology efficiency generalized entropy indexes for petroleum, coal, natural gas and fossil fuels. As a particular cases, we present the mean log deviation $(\alpha=0)$ and the Theil $(\alpha=1)$ indexes. It is interesting to notice how different is the behavior of the obtained generalized entropy indexes compared with the obtained Gini indices. Gini indexes seem to be smoother and they do not clearly reflect our intuition coming from the observed percentiles in Figures 1, 2, 3 and 4. For instance, the percentile band for petroleum consumption technology efficiency increases between 2005 and 2010. The increase in the percetile band indicates an increase in the global inquality of the technology efficiency spread. In this case, it is possible to notice that the corresponding generalized entropy indexes clearly indicate an increase in inequality between 2005 and 2010. For coal, the lower percentile band shrinks between 1995 and 2000 while the upper percentile band starts to increase between 2000 and 2005. It is important to notice that the corresponding Gini index shows a relatively smooth behavior, while the corresponding generalized entropy indices clearly indicates an asymmetry in change of the global technology efficiency distribution.

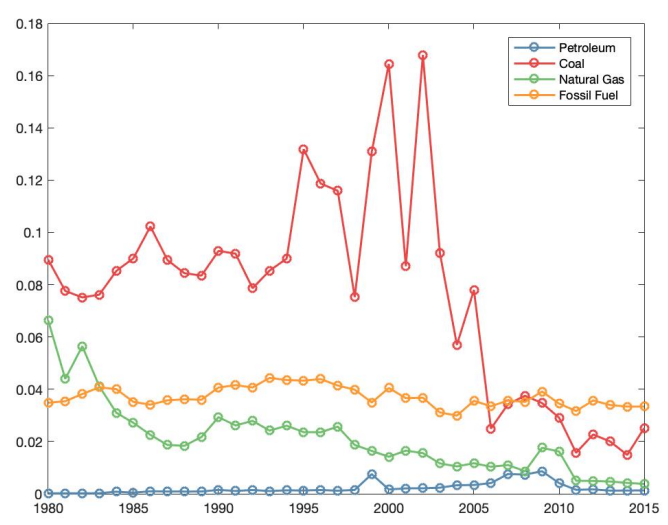

(a)

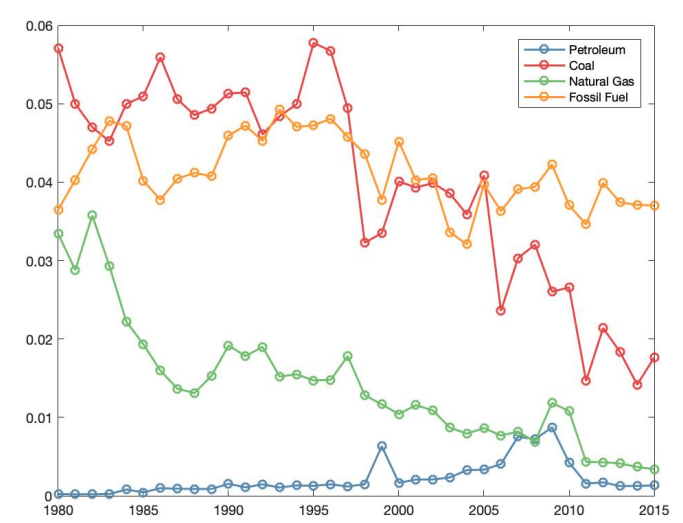

(b)

Figure 11. Technology efficiency generalized entropy indices for petroleum, coal, natural gas and fossil fuel using (a) $\alpha=0$ (mean log deviation index) and (b) $\alpha=1$ (Theil index).

In Figure 12, we present the technology efficiency Atkinson indexes for petroleum, coal, natural gas and fossil fuels. As particular cases, we present the Atkinson indices for $\epsilon=0.5$ and $\epsilon=1$. It is possible to notice a similarity between Atkinson indexes and generalized entropy indexes. Atkinson 
indexes for lower values of $\epsilon$ are quite similar to generalized entropy indexes for higher values of $\alpha$, and vice-versa. Either generalized entropy or Atkinson indexes become more sensitive to changes at the lower end of the technology efficiency distribution as, respectively, $\alpha$ approaches 0 and $\epsilon$ approaches 1. The same behavior is observed for the upper end of the technology efficiency distribution as, respectively, $\alpha$ approaches 1 and $\epsilon$ approaches 0 .

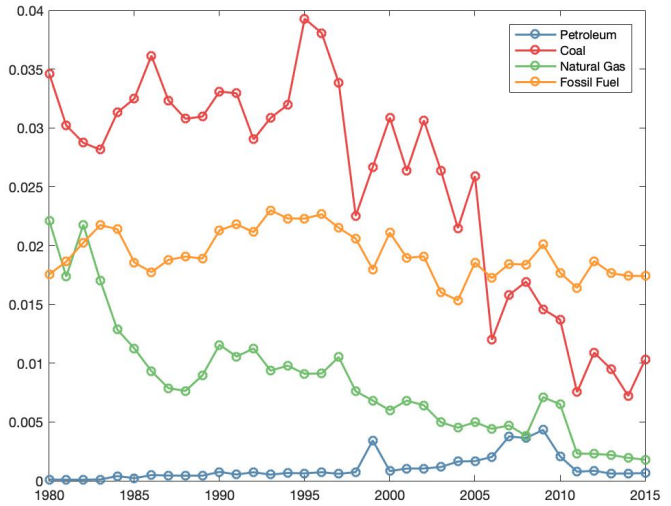

(a)

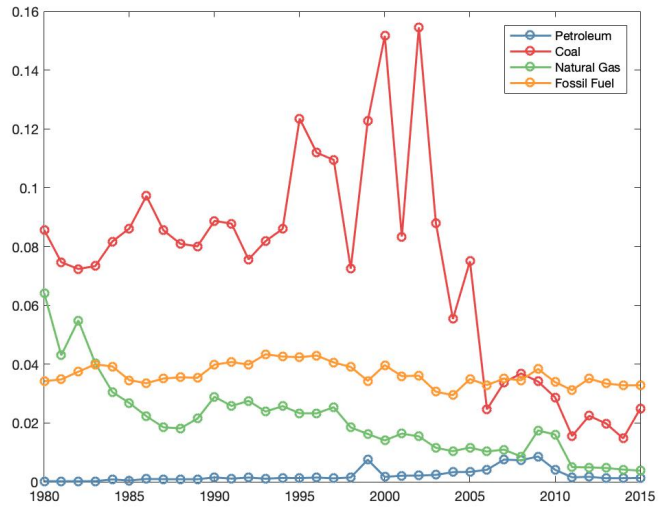

(b)

Figure 12. Technology efficiency Atkinson indexes for petroleum, coal, natural gas and fossil fuels using (a) $\epsilon=0.5$ and (b) $\epsilon=1$.

\subsection{Non-fossil fuel use global spread}

This subsection focus on global spreads in the use of non-fossil fuels. Figure 13, presents non-fossil fuel use for a sample of countries and the median, $5 \%$ percentile and $95 \%$ percentile for the entire set of considered countries. It is interesting to notice that the median value of use of non-fossil fuel increases comparing from 1980 to 2015. In addition, the lower percentile of these quantities seems to be more stable while the upper percentile presents a higher oscillation. The distance between the percentiles and the medians gives an intuitive idea of the global spread of use of non-fossil fuels along time. In the following paragraphs, we present our measures for global spread of use of non-fossils, based on Gini and generalized entropy indexes.

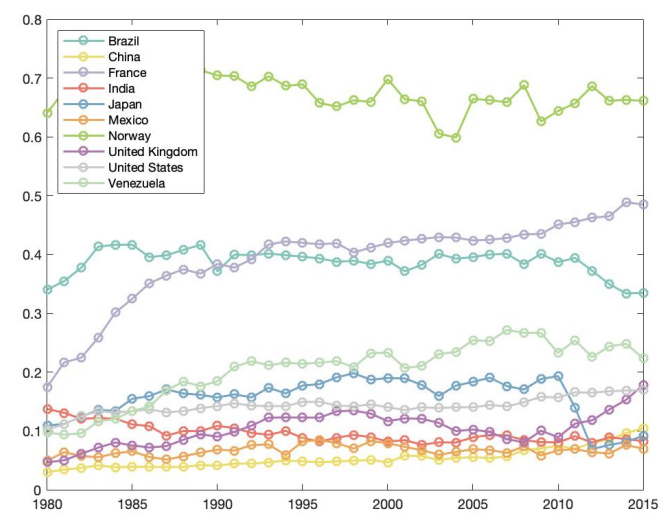

(a)

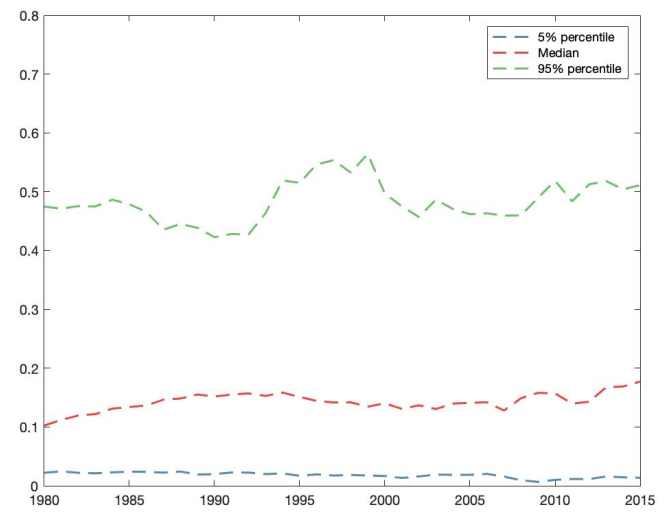

(b)

Figure 13. Non-fossil fuel use for (a) a sample of countries and (b) the median, $5 \%$ percentile and $95 \%$ percentile.

Non-fossil fuel Gini indexes are calculated from the corresponding Lorenz curves, as presented in Figure 14, for 1980 and 2015. These technology efficiency Gini indexes are close to zero, suggesting 
an almost homogeneous spread of technology efficiency among the different countries. In contrast, the non-fossil fuels Gini index is not close to zero, indicating a higher inequality of non-fossil fuel use between different countries.

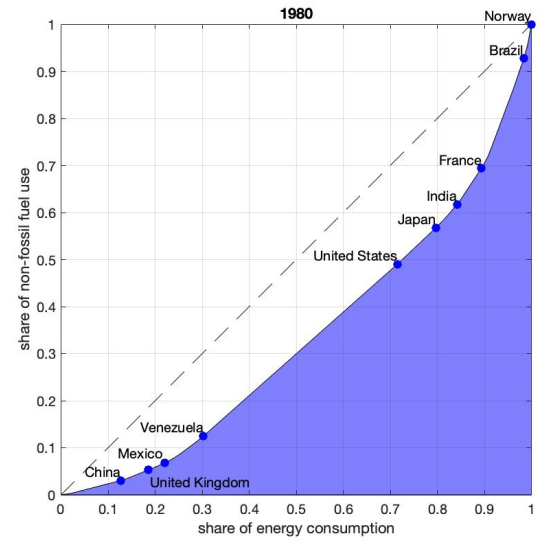

(a)

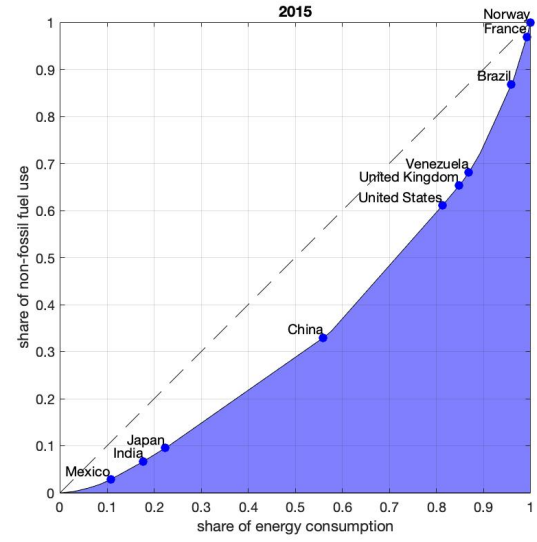

(b)

Figure 14. Lorenz curve of non-fossil fuels use in (a) 1980 and (b) 2015.

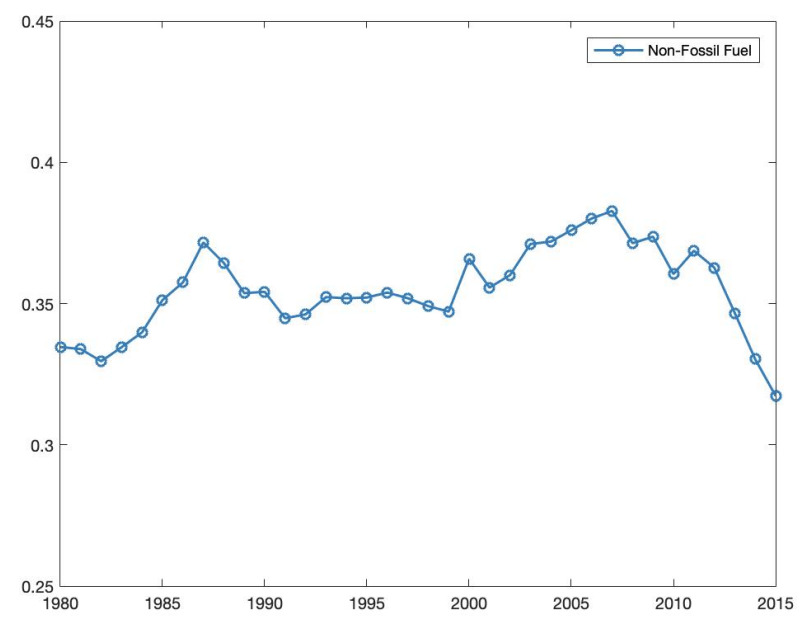

Figure 15. Non-fossil fuels Gini index.

In Figure 16, we present global non-fossil fuel use and its mean value among countries from 1980 to 2015. Notice that either global non-fossil fuel use or its mean value increase during the considered time interval. Moreover, non-fossil fuel use mean value among countries is higher than global non-fossil fuel use during the considered time interval due to the existence of countries with lower non-fossil fuel use with higher energy consumption. 


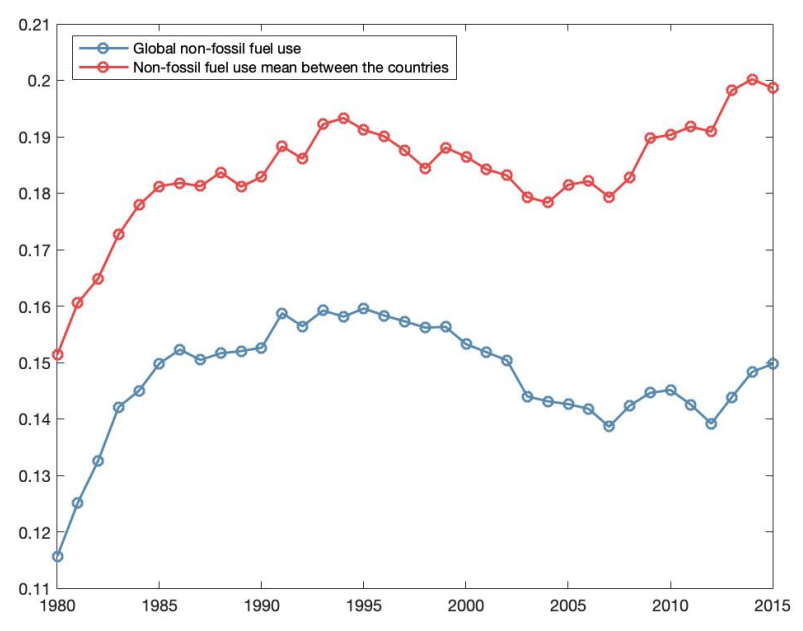

Figure 16. Global non-fossil fuel use and mean value among countries.

In Figure 17, we present the non-fossil fuel generalized entropy indexes for particular cases: the mean $\log$ deviation $(\alpha=0)$ and the Theil index $(\alpha=1)$. Notice the difference in behavior between generalized entropy indexes vs. Gini index. Intuitively, the generalized entropy indexes seem to better capture the percentiles behavior. For instance, in Figure 13b, between 1985 and 1995, there is a decrease in the $95 \%$ percetile. By inspection, it is also possible to notice a decrease in the same time period of the generalized entropy indexes in Figure 17. However, in Figure 15, the Gini index seems to increase in the same time period. Finally, in Figure 17, it is possible to notice that the mean log deviation index increases between 1980 and 2015, while the Theil index decreases. Consequently, inequality increases during the considered time period at the lower end of the non-fossil fuel use distribution. In contrast, inequality decreases during the considered time period at the upper end of the non-fossil fuel use distribution.

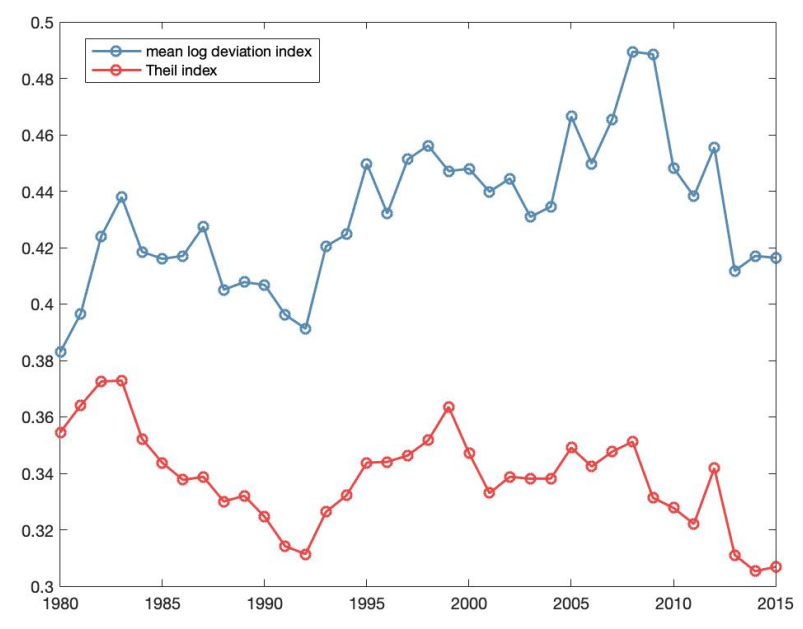

Figure 17. Non-fossil fuel use generalized entropy indices: mean log deviation index $(\alpha=0)$ and Theil index $(\alpha=1)$.

In Figure 18, we present non-fossil fuel Atkinson indexes for $\epsilon=0.5$ and $\epsilon=1.0$. Notice the similarity between the Atkinson indexes and generalized entropy indexes from Figure 17. Atkinson indices for lower values of $\epsilon$ are quite similar to the generalized entropy indexes for higher values of $\alpha$ and vice-versa. Either Atkinson or generalized entropy indexes for non-fossil fuels use confirm 
that, during 1980 and 2015, inequality increases at the lower end of the non-fossil fuel distribution and decreases at its upper end. In other words, the countries with higher non-fossil fuel use are getting more equally distributed in terms of non-fossil fuel participation in total energy consumption. In contrast, countries with lower non-fossil fuel use are getting more unequally distributed.



Figure 18. Non-fossil fuels use Atkinson indexes for $\epsilon=0.5$ (right axis) and $\epsilon=1.0$ (left axis).

Either technology efficiency and non-fossil fuels use are related to $\mathrm{CO}_{2}$ emissions, and they are influenced by government policies and other possible common factors. Consequently, we investigate the relationship between changes in the global spread of technology efficiency and changes in the global spread of non-fossil fuels use using the following linear regression models

$$
\begin{gathered}
G_{\varepsilon_{t}^{\text {fossil fuel }}}-G_{\varepsilon_{t-1}^{\text {fossil fuel }}}=a_{G}\left(G_{\rho_{t}}-G_{\rho_{t-1}}\right)+b_{G}, \\
E_{\varepsilon_{t}^{\text {fossil fuel }}}^{\alpha}-E_{\varepsilon_{t-1}^{\text {fossil fuel }}}^{\alpha}=a_{E}^{\alpha}\left(E_{\rho_{t}}^{\alpha}-E_{\rho_{t-1}^{\alpha}}^{\alpha}\right)+b_{E}^{\alpha}
\end{gathered}
$$

and

$$
A_{\varepsilon_{t}^{\text {fossil fuel }}}^{\epsilon}-A_{\varepsilon_{t-1}^{\text {fossil fuel }}}^{\epsilon}=a_{A}^{\epsilon}\left(A_{\rho_{t}}^{\epsilon}-A_{\rho_{t-1}}^{\epsilon}\right)+b_{A^{\prime}}^{\epsilon}
$$

where $a_{G}, b_{G}, a_{E}^{\alpha}, b_{E}^{\alpha}, a_{A}^{\epsilon}$ and $b_{A}^{\epsilon}$ are coefficients to be estimated. In Table 1, we present the estimated coefficients using ordinary least squares with the corresponding $p$-values, standard errors and $t$-statistics. In Table 2, we present some statistics of the estimated linear regression models: $R^{2}$, adjusted $R^{2}, p$-value, $F$-statistic and root mean squared error. Notice that the estimated values for $a_{G}, a_{E}^{\alpha}$ and $a_{A}^{\epsilon}$ are positive. Consequently, we can attest a positive relationship between changes in global spreads of the technology efficiency and changes in global spreads of non-fossil fuels use. In this respect, from Table 2 , it is also possible to notice that model 28 with $\alpha=0.0$ indicates a stronger positive relation than the model 28 with $\alpha=1.0$. This effect can be explained because the first model has a higher $R^{2}$, higher adjusted $R^{2}$, higher $F$-statistic, lower $p$-value and lower root mean squared error. Analogously, model 29 with $\epsilon=1.0$ indicates a stronger positive relation than model 29 with $\epsilon=0.5$, because the first model has a higher $R^{2}$, higher adjusted $R^{2}$, higher $F$-statistic and lower $p$-value. Since either generalized entropy or Atkinson indexes become more sensitive to changes at the lower end of technology efficiency and non-fossil fuels use distributions as $\alpha$ approaches 0 and $\epsilon$ approaches 1 , we conclude that attested positive relation between changes in global spreads of technology efficiency and changes in global spreads of non-fossil fuels use is higher at the lower end of the distributions. 
Table 1. Estimation of coefficients for the linear regression models 27, 28 and 29.

\begin{tabular}{ccccc}
\hline Coefficient & Estimate & $p$-value & Standard Error & $t$-statistic \\
\hline$a_{G}$ & 0.155 & 0.017 & 0.062 & 2.513 \\
$b_{G}$ & 0.001 & 0.033 & 0.001 & 2.230 \\
$a_{E}^{\alpha=0.0}$ & 0.084 & 0.002 & 0.025 & 3.408 \\
$b_{E}^{\alpha=0.0}$ & -0.000 & 0.782 & 0.000 & -0.279 \\
$a_{E}^{\alpha=1.0}$ & 0.077 & 0.207 & 0.059 & 1.288 \\
$b_{E}^{\alpha=1.0}$ & 0.000 & 0.853 & 0.001 & 0.186 \\
$a_{A}^{\epsilon=0.5}$ & 0.118 & 0.027 & 0.051 & 2.316 \\
$b_{A}^{\epsilon=0.5}$ & 0.000 & 0.933 & 0.000 & 0.085 \\
$a_{A}^{\epsilon=1.0}$ & 0.126 & 0.002 & 0.037 & 3.400 \\
$b_{A}^{\epsilon=1.0}$ & -0.000 & 0.777 & 0.000 & -0.286 \\
\hline
\end{tabular}

Table 2. Statistics for the linear regression models 27, 28 and 29.

\begin{tabular}{cccccc}
\hline Equation & $R^{2}$ & Adjusted $R^{2}$ & $p$-value & $F$-statistic & Root Mean Squared Error \\
\hline 27 & 0.161 & 0.135 & 0.017 & 6.320 & 0.003 \\
28 with $\alpha=0.0$ & 0.260 & 0.238 & 0.002 & 11.600 & 0.003 \\
28 with $\alpha=1.0$ & 0.048 & 0.019 & 0.207 & 1.660 & 0.004 \\
29 with $\epsilon=0.5$ & 0.140 & 0.114 & 0.027 & 5.360 & 0.002 \\
29 with $\epsilon=1.0$ & 0.259 & 0.237 & 0.002 & 11.600 & 0.003 \\
\hline
\end{tabular}

\section{Conclusions}

Our main contribution in this paper is the introduction of novel index measures for global spreads in primary energy consumption technology efficiency and non-fossil fuel use; these index measures are based on the Gini index and on generalized entropy indexes. In our empirical studies, these novel index measures proved to be effective, and could therefore provide helpful tools for policy planning and decision making regarding improvement of technology efficiency and the expansion non-fossil fuels use. In the empirical studies conducted in this paper, our novel index measures were able to capture interesting correlations or associations between several quantities of interest and their global spreads. Careful analysis of these and similar relations should prove important for strategic planing and allocation of capital and other resources by state entities, investment and energy firms, and other interested parties.

The empirical studies in this paper were conducted paying special attention to global spreads of technology efficiency and non-fossil fuels use. Nevertheless, methodology developed in this paper could also be applied to different studies, for example, comparing the development of different regions in a single country. Furthermore, studies of global spreads of fuel use could be extended to other primary energy sources, and also be used in the context of non-primary energy consumption. In future works, we plan to pursue these lines of research, always aiming for a cleaner and more sustainable world.

Author Contributions: Hellinton H. Takada developed the models, implemented the calculations and wrote the paper; Celma O. Ribeiro, Julio M. Stern and Oswaldo L. V. Costa provided scientific supervision. All authors have read and approved the final manuscript.

Acknowledgments: The authors would like to thank the anonymous reviewers for their valuable comments and suggestions to improve the quality of the paper. The authors are grateful for the support of POLI-USP, the Polytechnic School of the University of São Paulo; IME-USP, the Institute of Mathematics and Statistics of the University of São Paulo; and FAPESP, the State of São Paulo Research Foundation, through the Research Center for Gas Innovation under the grant 2014/50279-4.

Conflicts of Interest: The authors declare no conflict of interest.

\section{Abbreviations}


The following abbreviations are used in this manuscript:

BTU British Thermal Unit

EIA Energy Information Administration

MM Million Metric

US United States

\section{References}

1. BP. BP Statistical Review of World Energy; BP: London, UK, 2019.

2. United Nations. Emissions Gap Report; United Nations Environment Programme: Nairobi, Kenya, 2018.

3. International Energy Agency. World Energy Outlook 2018; International Energy Agency: Paris, France, 2018.

4. Awerbuch, S., Berger, M. Applying portfolio theory to EU electricity planning and policy-making. International Energy Agency/Emerging Energy Technologies: Paris, France, 2003.

5. Awerbuch, S. Portfolio-based electricity generation planning: policy implications for renewable and energy security. Mitig. Adapt. Strategies Glob. Chang. 2006, 11, 693-710.

6. Marrero, G. A., Ramos-Real, F. J. Electricity generation cost in isolated system: the complementarities of natural gas and renewables in the canary islands. Renew. Sustainable Energy Rev. 2010, 14, 2808-2818.

7. Losekann, L., Marrero, G. A., Ramos-Real, F. J., Almeida, E. L. F. Efficient power generating portfolio in Brazil: Conciliating cost, emissions and risk. Energy Pol. 2013, 62, 301-314.

8. Marrero, G. A., Puch, L. A., Ramos-Real, F. J. Mean-variance portfolio methods for energy policy risk management. Int. Rev. Econ. Finance 2015, 40, 246-264.

9. Costa, O. L. V., Ribeiro, C. O., Rego, E. E., Stern, J. M., Parente, V., Kileber, S. Robust portfolio optimization for electricity planning: An application based on the Brazilian electricity mix. Energy Econ. 2017, 64, 158-169.

10. Takada, H. H.; Stern, J. M.; Costa, O. L. V.; Ribeiro, C. O. Classical-equivalent Bayesian portfolio optimization for electricity generation planning. Entropy 2018, 20, 42.

11. Gini, C. Variabilità e Mutabilità: contributo allo studio delle distribuzioni e delle relazioni statistiche; Facoltá di Giurisprudenza della Regia Università di Cagliari, Tipografia di Paolo Cuppini: Bologna, Italy, 1912.

12. Heil, $\mathrm{M}$. T.; Wodon, Q. T. Inequality in $\mathrm{CO}_{2}$ emissions between poor and rich countries. Journal of Environment and Development 1997, 6, 426-452.

13. Heil, M. T.; Wodon, Q. T. Future inequality in $\mathrm{CO}_{2}$ emissions and the impact of abatement proposals. Environmental and Resource Economics 2000, 17, 163-181.

14. Groot, L. Carbon Lorenz curve. Resource and Energy Economics 2010, 32, 45-64.

15. Teng, F.; He, J.; Pan, X.; Zhang, C. Metric of carbon equity: carbon Gini index based on historical cumulative emission per capita. Adv. Clim. Chang. Res. 2011, 2, 134-140.

16. Lawrence, S.; Liu, Q.; Yakovenko, V. M. Global inequality in energy consumption from 1980 to 2010. Entropy 2013, 15, 5565-5579.

17. Soares, T. C.; Fernandes, E. A.; Toyoshima, S. H. The $\mathrm{CO}_{2}$ emission Gini index and the environmental efficiency: An analysis for 60 leading world economies. EconomiA 2018, 19, 266-277.

18. Shorrocks, A. F. The class of additively decomposable inequality measures. Econometrica 1980, 48, 613-625.

19. Bourguignon, F. Decomposable income inequality measures. Econometrica 1979, 47, 901-920.

20. Sauerbrei, S. Lorenz curves, size classification, and dimensions of bubble size distributions. Entropy 2010, 12, $1-13$.

21. Atkinson, A. B. On the measurement of inequality. J. Econ. Theory 1970, 2, 244-263.

22. US Energy Information Administration (EIA): International Energy Statistics. 